\title{
Improved sensitivity and temporal resolution in perfusion FMRI using velocity selective inversion ASL
}

\author{
Luis Hernandez-Garcia | Jon-Fredrik Nielsen | Douglas C. Noll \\ University of Michigan FMRI Laboratory, Ann Arbor, Michigan
}

\author{
Correspondence \\ Luis Hernandez-Garcia, University of \\ Michigan FMRI Laboratory, 2360 Bonisteel \\ Blvd., Ann Arbor, MI 48109-2108. \\ Email: hernan@umich.edu \\ Funding information \\ National Institute of Biomedical \\ Imaging and Bioengineering, Grant/ \\ Award Number: R21EB021562; National \\ Institutes of Health, Grant/Award Number: \\ R21EB021562
}

Purpose: This work aims to investigate the utility of velocity selective inversion pulses for perfusion weighted functional MRI.

Methods: Tracer kinetic properties of velocity selective inversion (VSI) pulses as an input function for an arterial spin labeling (ASL) experiment were characterized in a group of healthy participants. Numerical simulations were conducted to search for a robust set of timing parameters for FMRI time series acquisition with maximal signal to noise ratio efficiency. The performance of three VSI pulse sequences with different timing parameters was compared with a pseudocontinuous ASL sequence in a simple FMRI experiment conducted on healthy participants.

Results: The fit to the tracer kinetic model yielded arterial CBV of $1.24 \% \pm 0.52 \%$ and $0.45 \pm 0.11 \%$ and perfusion rates of $60.8 \pm 32.3$ and $34.4 \pm 5.4 \mathrm{~mL} / \mathrm{min} / 100 \mathrm{~g}$ for gray and white matter, respectively. Bolus arrival times were estimated as $75.7 \pm$ $21 \mathrm{~ms}$ and $349 \pm 78 \mathrm{~ms}$ for gray and white matter, respectively. The FMRI experiments showed that VSI pulses yield comparable sensitivity to PCASL with similar timing parameters $(T R=4 \mathrm{~s})$. However, VSI pulses could be used at a faster acquisition speed (TR $=3 \mathrm{~s}$ ) and were more sensitive to neuronal activity than PCASL pulses, as evidenced by the $31 \%$ higher $\mathrm{Z}$ scores obtained on average in the active regions.

Conclusion: VSI pulses can be very beneficial for perfusion weighted functional MRI because of their tracer kinetic characteristics, which allow a faster acquisition rate while maintaining an efficient labeling input function.

\section{K E Y W O R D S}

arterial spin labeling, functional MRI, velocity selective inversion, VSI, ASL, brain, perfusion, bolus arrival time

\section{1 | INTRODUCTION}

Arterial spin labeling (ASL) allows for quantitative imaging of perfusion without the use of exogenous tracers. ${ }^{1}$ In addition to baseline perfusion imaging, ASL techniques can also be used to carry out functional MRI experiments, as changes in perfusion are well known to be related to changes in brain activity. ASL-based FMRI is generally challenged by low SNR, although it offers significant advantages in terms of its stability and its ability to produce a quantifiable measurement of a physiological parameter. Indeed, ASL based FMRI was found to be more stable across subjects than BOLD or 
even calibrated $\mathrm{BOLD}^{2-4}$ and many longitudinal FMRI studies have used ASL acquisitions (for examples, see refs ${ }^{5-8}$ ).

Velocity and acceleration selective pulses have been recently shown to be an attractive option for ASL perfusion imaging because of potential gains in SNR and decreased sensitivity to bolus arrival time variations. ${ }^{9-14}$ While typical ASL labeling schemes target inflowing arterial blood spins based on their spatial position relative to the tissue of interest (e.g., pseudocontinuous ASL, or PCASL, pulses label spins as they cross a plane upstream of the tissue of interest), velocity selective labeling, on the other hand, targets moving spins regardless of their location. As a result, the time elapsed between the spins getting labeled and arriving at the capillary bed is virtually removed. Previous work has demonstrated that velocity selective saturation pulses can thus eliminate bolus arrival time effects. ${ }^{11}$ Furthermore, velocity selective inversion (VSI) pulses yield greater labeling efficiency ${ }^{14,15}$ Because velocity selective labeling ASL can be potentially acquired faster than continuous ASL, this could result in an additional SNR boost. As a result, we hypothesize that VSI-based ASL acquisitions will produce images with SNR greater than PCASL, and with reduced sensitivity to arrival time variations.

Thus, one goal of this work was to test the utility of VSI pulses for perfusion-based functional brain imaging and to optimize the timing parameters of ASL image time series acquisition, which has not been previously investigated. The optimized ASL should achieve higher temporal resolution and higher SNR in ASL imaging, by collecting more images per unit time.

In this work, we first measured and characterized the uptake of the input function generated by VSI pulses experimentally. We aimed to verify theoretical predictions about the efficiency of the labeling pulses under experimental conditions and about the tracer kinetics model of the ASL signal generated by VSI pulses. We also aimed to obtain estimates of the width and amplitude of the input bolus to design the timing parameters of the FMRI sequence. We then tested the performance of VSI-ASL against the more standard PCASL method in a simple perfusion weighted FMRI experiment. We also compared different timing parameters for the VSI acquisition to investigate the sensitivity, SNR, and functional contrast-to-noise ratio (CNR) of the pulse sequence.

\section{$2 \mid$ METHODS}

\section{1 | Characterization of the VSI input function: Experiments}

In the first experiment, five healthy volunteers, aged between 40 and 56 years were scanned using a 3T MR750 scanner (GE Medical Systems, Waukesha, WI) and a 32-channel receive-only coil (Nova Medical, Wilmington, MA). As depicted in Figure 1, the acquisition scheme consisted of a 3D stack of spirals acquisition preceded by an ASL preparation as in Nielsen and Hernandez-Garcia. ${ }^{16}$ In this case, the ASL preparation was done with a VSI pulse, ${ }^{15}$ a variable postlabeling delay, and an optional velocity selective arterial saturation pulse for arterial suppression $50 \mathrm{~ms}$ before acquisition. No background suppression pulses were used. Label uptake curves were collected by varying the delay between labeling and acquisition, to estimate the following unknown parameters: (1) bolus arrival time, (2) perfusion rate, and (3) arterial blood volume fraction in both the gray and white matter.

The VSI pulses were generated using the method described by Qin et al. Specifically, The VSI pulses (see Supporting Information Figure S1, which is available online) consisted of a train of nine $20^{\circ}$ hard pulse segments



F I G U RE 1 Schematic diagram of an ASL sequence using Velocity Selective Inversion pulses for labeling. Optional vascular suppression consisting of BIR-8 Velocity Selective Saturation pulses can be included immediately before acquisition. Image acquisition is carried out using 3D stack of spirals 
(81.25 mG for $160 \mu \mathrm{s})$ separated by 6-ms gaps. Two refocusing hard pulses (117 $\mathrm{mG}$ for $1 \mathrm{~ms}$ ) were inserted in those gaps with an MLEV phase pattern. ${ }^{14,17}$ Velocity encoding was done along the $\mathrm{Z}$ axis by inserting triangular gradient pulses $(\mathrm{Gmax}=2 \mathrm{G} / \mathrm{cm}, 300 \mu \mathrm{s}$ ramp, 200$\mu$ s gap between pulses) of alternating sign between RF pulses. The nonselective case was carried out by using gradient pulses of the same sign. The total duration of the pulse train was $49.4 \mathrm{~ms}$ The arterial suppression pulses were based on a symmetric BIR-8 velocity selective saturation scheme, as shown in Supporting Information Figure S2. ${ }^{18,19}$ A Bloch simulation of the velocity profile of both of these pulses is shown on the right side of Supporting Information Figure S3. The Bloch simulations used $\mathrm{T} 1$ of $1700 \mathrm{~ms}$, and T2 of $150 \mathrm{~ms} .{ }^{15,20}$ The velocity profiles of both pulses can be seen in greater detail in Supporting Information Figure S3. We note that the cutoff velocity for the velocity saturation pulse was set to $0.7 \mathrm{~cm} / \mathrm{s}$, and that the efficiency of the velocity selective inversion pulse varies with velocity, as expected. The theoretical mean labeling efficiency (defined as the magnetization difference between velocity selective and nonselective cases, relative to the relaxed magnetization) is $84 \%$ for the range between 5 and $80 \mathrm{~cm} / \mathrm{s}$.

The spiral image acquisition parameters were: matrix size $=128 \times 128 \times 16$, voxel size $=0.18 \times 0.18 \times 0.6 \mathrm{~cm}$, $\mathrm{BW}=85 \mathrm{kHz}, \mathrm{TR}=4000 \mathrm{~ms}, \mathrm{TE}=4.5 \mathrm{~ms}$, nominal flip angle $=30^{\circ}$, No. interleaves $=2$, No. of averages $=10(5$ control and 5 labeled), two dummy scans. The total scan time was $88 \mathrm{~s}$.

To estimate the uptake rate of the VSI input function into the extravascular space and to characterize its shape, $13 \mathrm{im}$ ages were collected with different post inversion delays from 200 to $2600 \mathrm{~ms}$ with no arterial suppression. The experiment was then repeated with arterial suppression pulses (BIR-8 velocity selective saturation) turned on. No VSI or arterial suppression pulses were applied in the first two images of the time series to collect reference images. VSI pulses were applied in the remaining eight images, alternating between selective and nonselective pulses.

Images were reconstructed, realigned, and smoothed with a Gaussian kernel (full width at half $\max =6 \mathrm{~mm}$ ). The nonselective images at each post inversion delay were used to fit an inversion recovery curve and estimate the longitudinal relaxation rate, and the inversion efficiency of the VSI pulses in the stationary tissue. The resulting R1 maps were used to segment gray matter and white matter regions.

The ASL signal at each voxel was calculated as the percentage signal difference between the magnitudes of the selective and nonselective images, relative to the reference images (i.e., no prep. pulses). The average ASL signal as a function of the post inversion delay was calculated for white and gray matter separately for each subject.

\section{2 | Characterization of the VSI input function: Theory}

A two-compartment model (vascular and extra vascular) of the ASL signal (without arterial suppression) was constructed to fit the observed data as a function of the post inversion delay, as follows. ${ }^{21,22}$ The extravascular compartment's longitudinal magnetization is described by a modified Bloch equation:

$$
\frac{d M_{z}}{d t}=\left(M_{z}^{0}-M_{z}(t)\right) \cdot R_{1}+f \cdot M_{a}(t)-\frac{f}{\lambda} \cdot M_{z}(t)
$$

where $M_{z}(t)$ and $M_{a}(t)$ are the longitudinal magnetization of tissue and arterial blood, respectively. R1 is the tissue's relaxation rate. Perfusion rate is captured by $f$, and $\lambda$ is the blood:brain partition coefficient. The arterial compartment can be modeled by a simple inversion-recovery equation, following the VSI pulse:

$$
M_{a}(t)=M_{a}^{0}\left(1-2 \cdot \alpha \cdot e^{-t \cdot R 1_{a}}\right)
$$

Here, $\alpha$ is the efficiency of the VSI pulse, and $R_{1 a}$ is the longitudinal relaxation rate of arterial blood. For the arterial blood, $\alpha$ was assumed to be 0.15 in the velocity selective case and 0.7 in the nonselective case.

To capture the bolus arrival time (BAT), defined as the time it takes the uninverted spins in the velocity selective case to reach the extra-vascular compartment, we modified the arterial magnetization input into the tissue, such that in the velocity selective case, the early part of the input is inverted, but after a delay, the noninverted spins arrive into the tissue compartment. Thus, the arterial input function is made up of both inverted and un-inverted spins, in varying proportion. Specifically, the new arterial magnetization function is.

$$
M_{a}(t)=B(t) \cdot M_{a, \text { sel }}(t)+(1-B(t)) \cdot M_{a, \text { nonsel }}(t)
$$

Where $B(t)$ is the proportion of spins in the arterial compartment that is un-inverted by the VSI pulse. $B(t)$ is a sigmoid function whose inflection point happens at the bolus arrival time. It was constructed by integrating a Gaussian distribution function, whose mean was the bolus arrival time, and its standard deviation was fixed to $100 \mathrm{~ms}$, as an approximation of the distribution.

Finally, the observed signal, $S(t)$, is a mix of the vascular and extravascular compartments. It can be written as.

$$
S(t)=C B V_{a} \cdot M_{a}(t)+\left(1-C B V_{a}\right) \cdot M_{z}(t)
$$

where $C B V_{a}$ is the arterial blood volume fraction.

As in most ASL experiments, the VSI experiment consists of two acquisitions preceded by velocity selective and 
nonselective inversion pulses. In the nonselective case, the initial conditions for arterial and extravascular compartments are $M_{a}(0)=1-2 \cdot \alpha$ and $M_{z}(0)=1-2 \cdot \alpha_{e}$, respectively $(\alpha$ and $\alpha_{e}$ denote separate inversion efficiency for vascular and extravascular spins). In the velocity selective case, the arterial magnetization in the extravascular compartment is still inverted, but the arterial magnetization is "lightly" saturated by the VSI pulses and $M_{a}(0)=1-\alpha$.

We used a nonlinear least squares algorithm to estimate (i) bolus arrival time, (ii) perfusion rate, (iii) arterial blood volume fraction, and (iv) arterial inversion efficiency from both gray and white matter using the unsuppressed uptake curves. We fixed the inversion efficiency of the arterial blood to 0.58 for the arterial spins for the white matter data, based on the gray matter results (we found that the white matter data were too noisy to fit all the parameters at once. To reduce to problem size, we fixed the value of the inversion efficiency to the mean of the gray matter value). We assumed $\mathrm{T} 1$ relaxation of arterial blood to be $1700 \mathrm{~ms}^{23}$

\section{3 | Numerical optimization of timing parameters}

Using Equations 1-4, we simulated the longitudinal magnetization in a voxel with the above VSI pulse sequence with multiple TRs (from 2-5 s), and postlabeling delays (0.5-2 s) to design a VSI sequence for fast perfusion-weighted FMRI that maximizes the SNR of the ASL signal for a given total imaging time. We used the same two-compartment model as before, and the following parameters, based on the estimates from the uptake curves above: perfusion rate $=60 \mathrm{~mL} /$ $\min / 100 \mathrm{~g}$, arterial blood volume $=1 \%$, VSI bolus width $=$ $2600 \mathrm{~ms}$, tissue T1 = $1400 \mathrm{~ms}$, arterial T1 = $1700 \mathrm{~ms}$, inversion efficiency $=80 \%$. We assumed that 3D image acquisition takes $600 \mathrm{~ms}$ (experimentally, the acquisition time for 3D stack of spirals was $521 \mathrm{~ms}$ ). Stationary spins were assumed to be completely saturated by the end of the image acquisition window. We used this framework to calculate the ASL signal (difference between selective and nonselective images). The relative SNR of the signal per unit of time was calculated by scaling the ASL signal by the square root of the number of images collected in that unit of time (or one over the square root of TR).

\section{$2.4 \quad$ Eddy current tests}

In a separate experiment, we tested the eddy current artefacts due to the VSI pulses described in the original implementation study by Qin et al, ${ }^{24}$ by repeating the above uptake experiment on an FBIRN gel phantom. ${ }^{25}$ We examined the signal differences between velocity selective and nonselective acquisitions in terms of the differences introduced into the magnitude and the phase of the images. We calculated the root mean square phase and magnitude differences, separately, as a function of the post inversion delay.

\section{5 | FMRI experiments}

Healthy subjects ( $\mathrm{N}=6$, ages from 40 to 56 years) were scanned using a 3.0T MR750 scanner. The subjects were instructed to tap their fingers of their right hand for $20 \mathrm{~s}$ while being stimulated with an $8-\mathrm{Hz}$ flashing checkerboard pattern. The stimulus was repeated 5 times, preceded by $40 \mathrm{~s}$ of inactivity (total time $=300 \mathrm{~s}$ ). This experimental paradigm was repeated four times while the subjects were scanned with the following four ASL sequences, using parameters based on simulation: (1) PCASL labeling for $1600 \mathrm{~ms}$, followed by $1800 \mathrm{~ms}$ post inversion delay (PID), $\mathrm{TR}=4000 \mathrm{~ms}$; (2) VSI label followed by $1800 \mathrm{~ms}$ PID, TR $=4000 \mathrm{~ms}$; (3) VSI label followed by $1800 \mathrm{~ms}$ PID, TR = $3000 \mathrm{~ms}$; and (4) VSI label followed by $1200 \mathrm{~ms}$, TR = $2000 \mathrm{~ms}$ As before, acquisition in all four cases was done with a $3 \mathrm{D}$ stack of spirals sequence $(64 \times 64 \times 16$ matrix, $0.344 \times 0.344 \times 0.6 \mathrm{~cm}$ voxel size $)$. No background or arterial suppression pulses were used. Off resonance correction for the PCASL pulses was applied after a calibration experiment, as recommended in Jahanian et al. ${ }^{26}$

The time series was reconstructed, motion corrected, smoothed with $6 \mathrm{~mm}$ Gaussian kernel and surround-subtracted in time. Linear regression was used to detect and estimate stimulus related activation, including nuisance regressors identified using the CompCor technique. ${ }^{27} \mathrm{Z}$ score maps corresponding to the activation were calculated from the resulting estimates. The number of voxels with a $\mathrm{Z}$ score greater than 3 within the brain were tabulated for each case.

Spatial noise was estimated as the standard deviation of the temporal mean magnitude of pixels outside the brain. Temporal noise was estimated as the temporal standard deviation averaged over all the pixels inside the whole brain (without removal of task or drift effects). Spatial and temporal SNR were defined as the mean ASL signal inside the brain divided by the spatial and temporal noise standard deviations, respectively. Contrast-to-noise ratio (CNR) can be inferred from the $\mathrm{Z}$ scores, which are derived from the $\mathrm{T}$ statistic; in this case, a ratio of the activation size estimate over the estimate of its variance and accounting for the number of measurements as degrees of freedom.

\section{3 | RESULTS}

\section{1 | Characterization of the VSI input function and pulse sequence optimization}

Figure 2 shows the simulated longitudinal magnetization $(\mathrm{Mz})$ of the arterial compartment and of the extravascular compartment following the VSI pulses, using the constants 
Simulated Longitudinal Magnetization in VSI experiment

F I G URE 2 Bloch simulated magnetization for both arterial and extravascular compartments after a VSI pulse, based on Equations ${ }^{1-4}$. On the top, a nonselective pulse inverts the magnetization in both moving (vascular) and quasi-stationary (extravascular) spins. The middle panel shows the velocity selective case, in which only the quasi-stationary (extravascular) spins are inverted. Finally, the bottom panel shows the observed difference between the observed signals in the velocity selective and nonselective cases as a function of time after the pulse is applied. If magnitude-only images are used, as is routine in MR imaging, one should observe a sign reversal in the early part of the curve
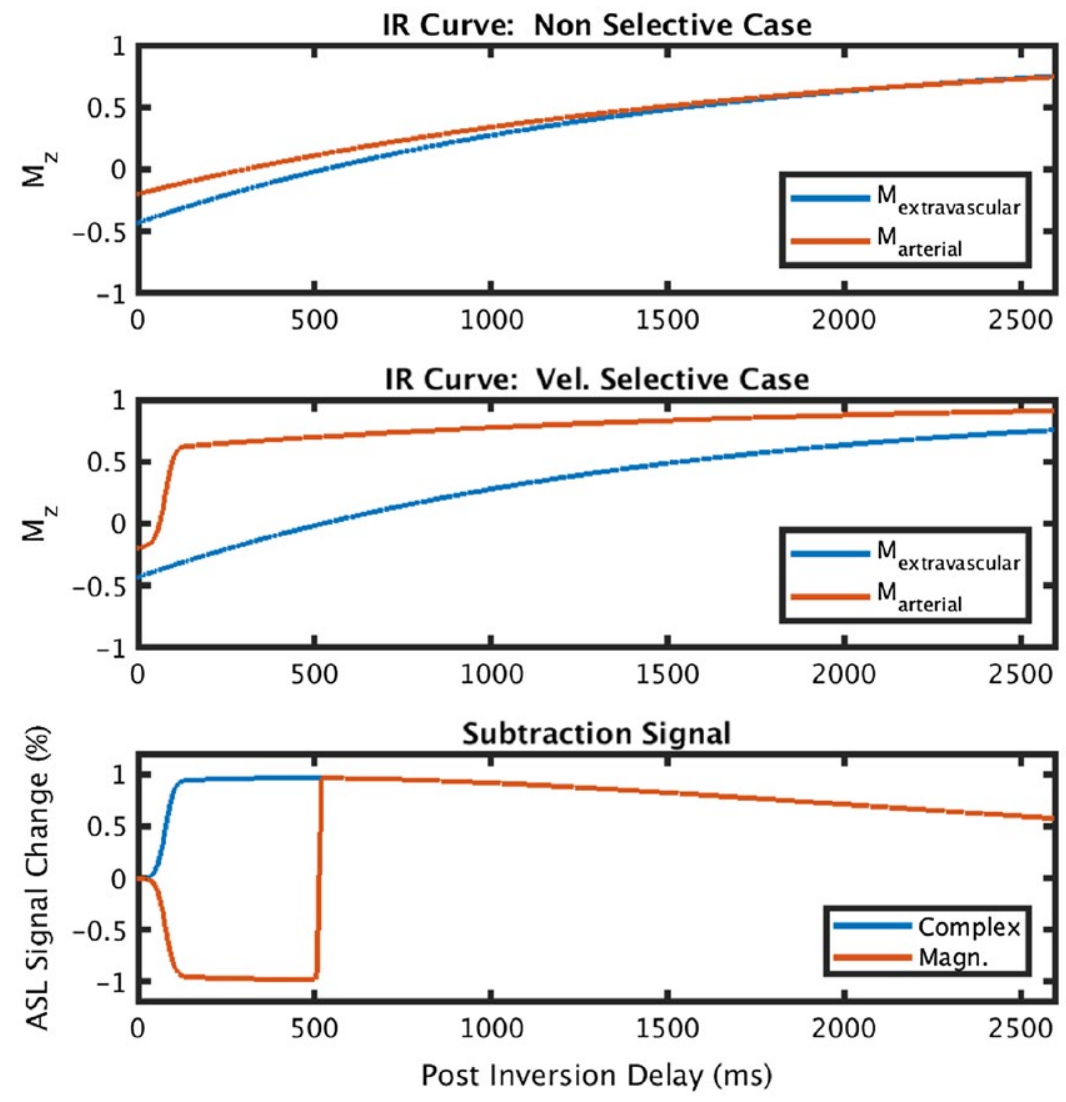

extracted from gray matter voxels in a representative subject. In the nonselective case, shown in the top panel, both the arterial and extravascular spins are inverted with 59\% and $72 \%$ efficiency, respectively, and relax at their corresponding T1 rates of $1700 \mathrm{~ms}$ and $1400 \mathrm{~ms}$, respectively. In the velocity selective case, shown in the middle panel, the arterial compartment is assumed to be tipped down only $15 \%$. However, when acquiring images immediately after the inversion pulse, the signal is dominated by the slow moving spins in the extravascular compartment and in the arterioles, which are inverted by the pulse. There is a short delay before the un-inverted spins move into the arterioles, capillaries and extravascular space. Consequently, the model includes a delay (or "bolus arrival time") before the un-inverted spins entered the compartment.

Finally, the ASL signal is shown in the bottom panel as the difference between the total magnetization in the selective and the nonselective cases. If the subtraction is performed using complex images, then its value should essentially reflect the concentration of labeled spins in the voxels as a function of the post inversion delay (shown in blue). Note, however, that ASL images are typically constructed by subtracting magnitude images and this results in the sign flip in the early part of the curve, before the labeled arterial spins have reached the null point of their recovery and still have negative longitudinal magnetization. This would rarely affect the use of VSI-ASL in practice, because postlabeling delays are typically longer than $1 \mathrm{~s}$.

The same images from the uptake experiments (using variable post inversion delays) were first used to estimate $\mathrm{T} 1$ relaxation and inversion efficiency. The average $\mathrm{T} 1 \mathrm{re}-$ laxation rates estimated from the unsubtracted, nonselective images as a function of the post inversion delay were $1475 \pm$ 11 and $892 \pm 22 \mathrm{~ms}$ for gray and white matter, respectively. From the same nonlinear least-squares fit, the average inversion efficiency of the stationary tissue was $71.6 \% \pm 1.0 \%$ and $73.9 \% \pm 1.9 \%$ in the gray and white matter, respectively.

ASL images collected at different times after the VSI pulse can be seen in Figure 3. ASL time courses as a function of the post inversion delay were extracted from gray and white matter, and averaged separately across all participants, as shown in Figure 4. We note that early images after the VSI pulse clearly show the arterial network as a negative signal. We also note that the vasculature is not visually apparent (Figure 3) when the arterial suppression pulse was used.

As predicted by simulations in Figure 2, the ASL images have negative value initially, and the sign of the ASL image changes around between 600 and $800 \mathrm{~ms}$ after the inversion pulse. The ASL signal decays afterward. This is consistent 


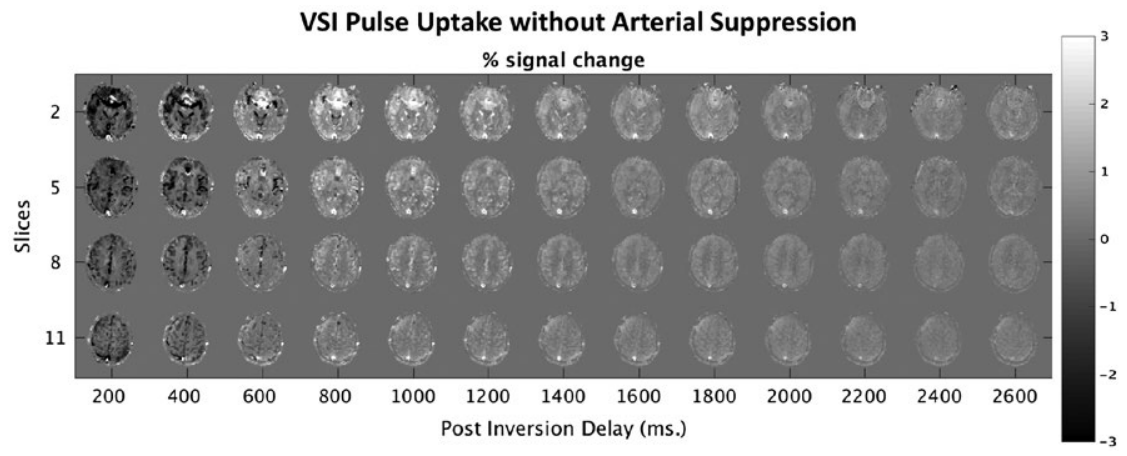

F I G URE 3 Subtraction images collected at different times after the application of a VSI pulse. The images are computed as $\%$ signal difference between selective and nonselective cases, relative to a reference image without any preparation pulses. Each column shows four slices from the collected volume. Each row represents a single slice collected at different post inversion delays. The images were acquired without using vascular suppression. Supporting Information Figure S4 shows the results from the same experiment using a BIR- 8 velocity selective saturation pulse for vascular suppression. These results are consistent with Figure 2
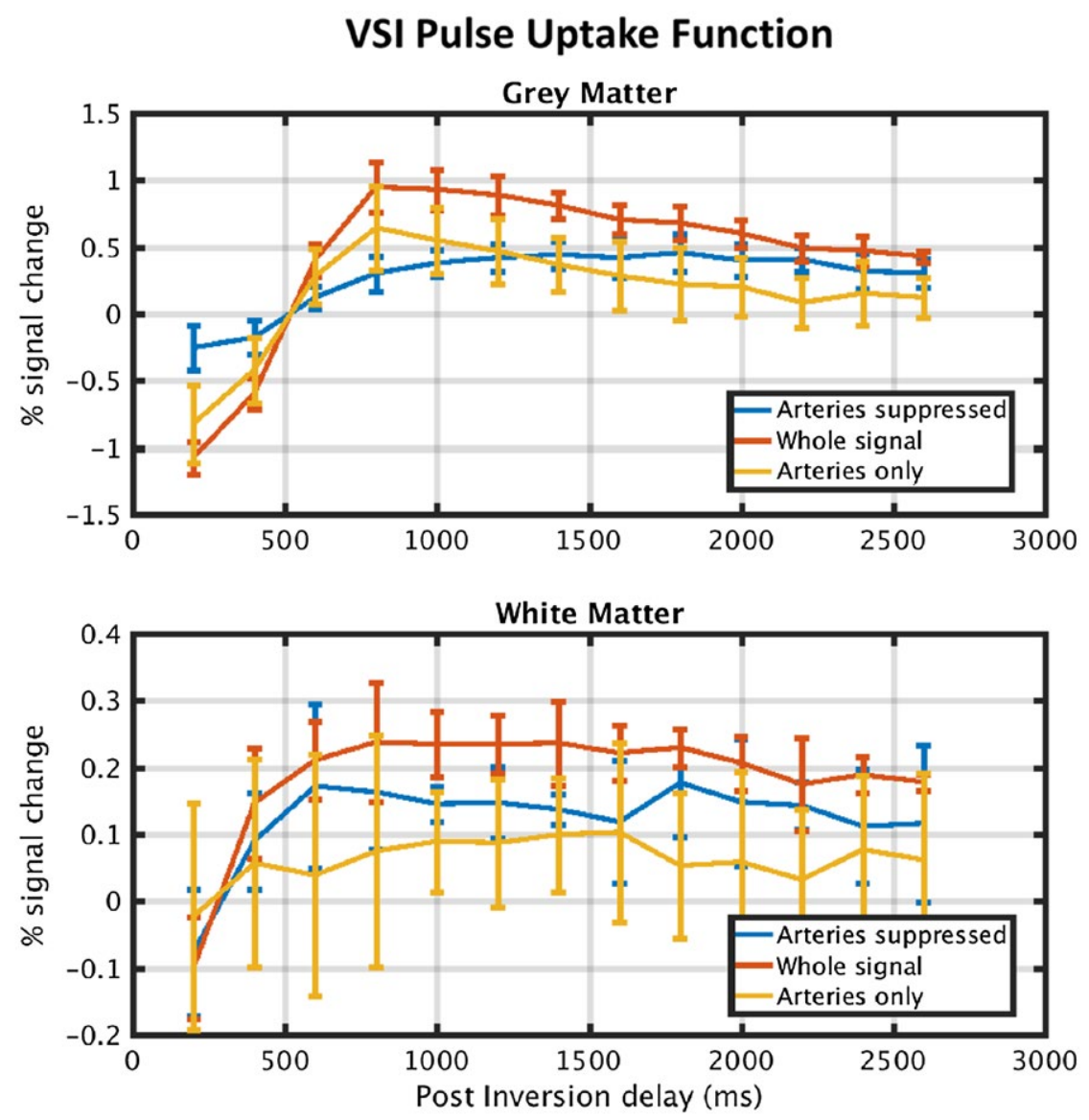

F I G URE 4 Percent signal change observed after a delay following the application of a VSI pulse (see Figure 3). These time courses were extracted from gray and white matter regions separately and averaged across all participants

with the labeling pulse inverting the magnetization in the arteries, which then relaxes back to the positive axis, and with the fact that the ASL images were calculated as a subtraction of magnitude images.

The proposed two-compartment model fit to the unsuppressed time courses can be seen in Figure 5 (in agreement with the predicted curves in Figure 2). The parameter estimates obtained from the fitting procedures are shown in Table 1 To summarize, the fit to the tracer kinetic model yielded arterial CBV of $1.2 \% \pm 0.52 \%$ and $0.45 \pm 0.11 \%$ and perfusion rates of $60.8 \pm 32.2$ and $34.4 \pm 5.4 \mathrm{~mL} / \mathrm{min} / 100 \mathrm{~g}$ for gray and white matter, respectively. Bolus arrival times 
Grey Matter
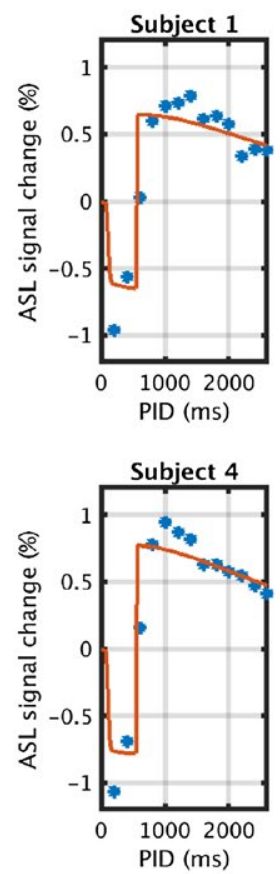
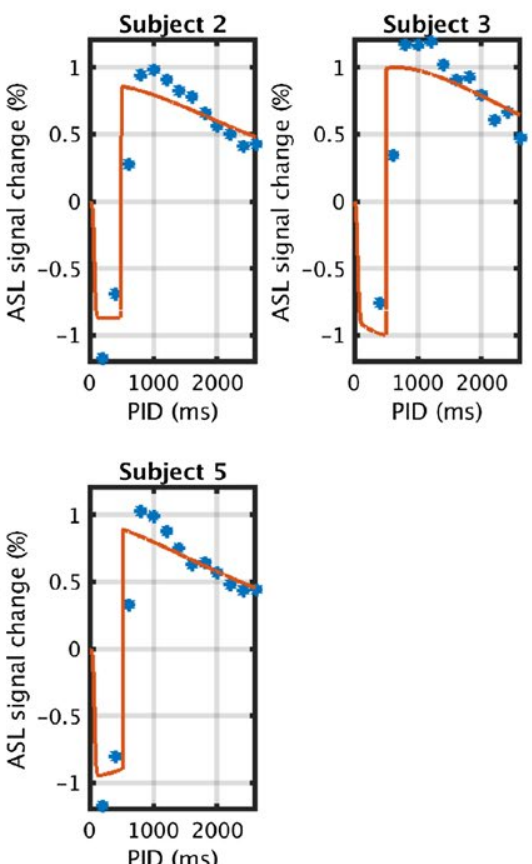

White Matter
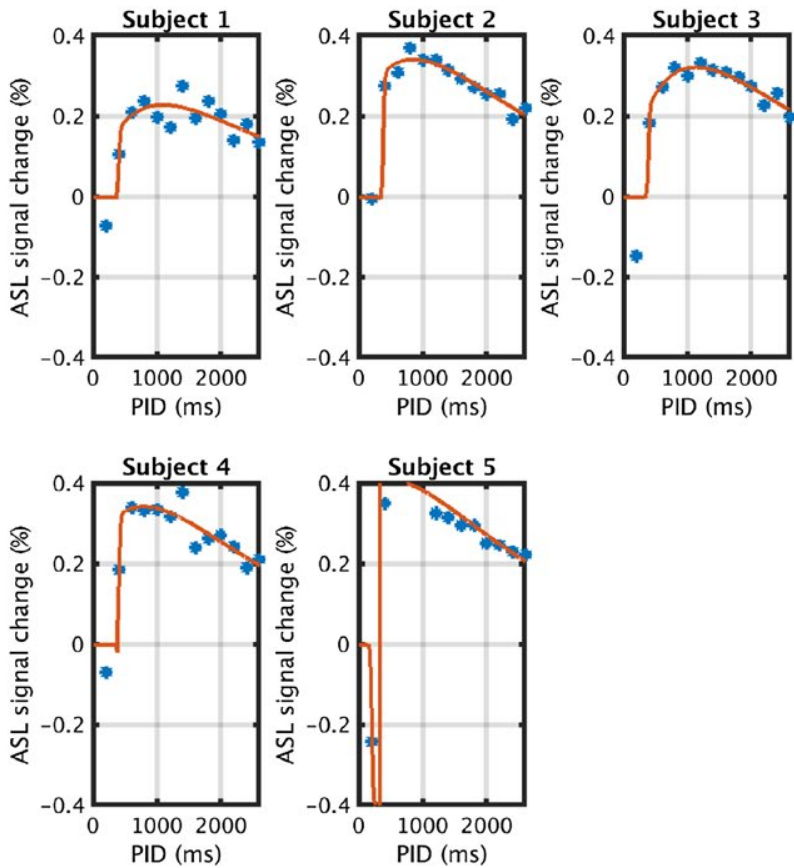

F I G U RE 5 Model fit of the VSI kinetic function to the observed experimental data for each subject, indicating good agreement between the two

TA B L E 1 Hemodynamic parameter fits from the curves shown in Figure 5

\begin{tabular}{|c|c|c|c|c|c|c|c|}
\hline \multicolumn{4}{|l|}{ White matter } & \multicolumn{4}{|l|}{ Gray matter } \\
\hline 59 & 0.28 & 30.8 & 394 & 69 & 0.58 & 33.2 & 100 \\
\hline 59 & 0.50 & 34.4 & 376 & 50 & 1.8 & 76.1 & 62.6 \\
\hline 59 & 0.37 & 44.7 & 383 & 50 & 1.8 & 117.4 & 50.0 \\
\hline 59 & 0.57 & 30.0 & 194 & 59 & 1.3 & 41 & 65.5 \\
\hline $59 *$ & $0.45 \pm 0.11$ & $34.4 \pm 5.4$ & $349 \pm 77.5$ & $59+/ 8.8$ & $1.2 \pm 0.52$ & $60.8 \pm 32$ & $75.6 \pm 20.6$ \\
\hline
\end{tabular}

*Inversion efficiency estimates assumed to be the same as the grey matter.

were estimated as $75.6 \pm 21 \mathrm{~ms}$ and $349 \pm 77 \mathrm{~ms}$ for gray and white matter, respectively. The inversion efficiency in the arterial blood was estimated to be $59 \% \pm 8.8 \%$ in the gray matter. This efficiency was assumed to be the same for the white matter compartment, as well.

Figure 6 shows the simulated evolution of the longitudinal magnetization of both the arterial and the extravascular compartments of a theoretical gray matter voxel, when imaged with a VSI perfusion weighted FMRI sequence time series (at rest). The arterial compartment is inverted by the nonselective pulses, but only partially tipped by the velocity-selective pulses. The extravascular compartment (stationary and slow moving spins) becomes inverted in both cases. We also note that in our simulation model, the 3D image acquisition was assumed to saturate all the spins in the imaging slab, but that the arterial spins are replaced before the next labeling period. We calculated the expected ASL signal (\% signal change) after two cycles, when the system reaches an equilibrium, and its relative SNR efficiency (the SNR within a fixed scanning period, or SNR per unit of time).

Figure 7 shows the expected ASL signal (top two panels) and its SNR efficiency (bottom two panels) as a function of TR and post inversion delay. The left side shows the signals from the whole voxel, whereas the right side shows only the extravascular compartment (i.e., the case when arterial suppression 


\section{Simulation of VSI time series FMRI}

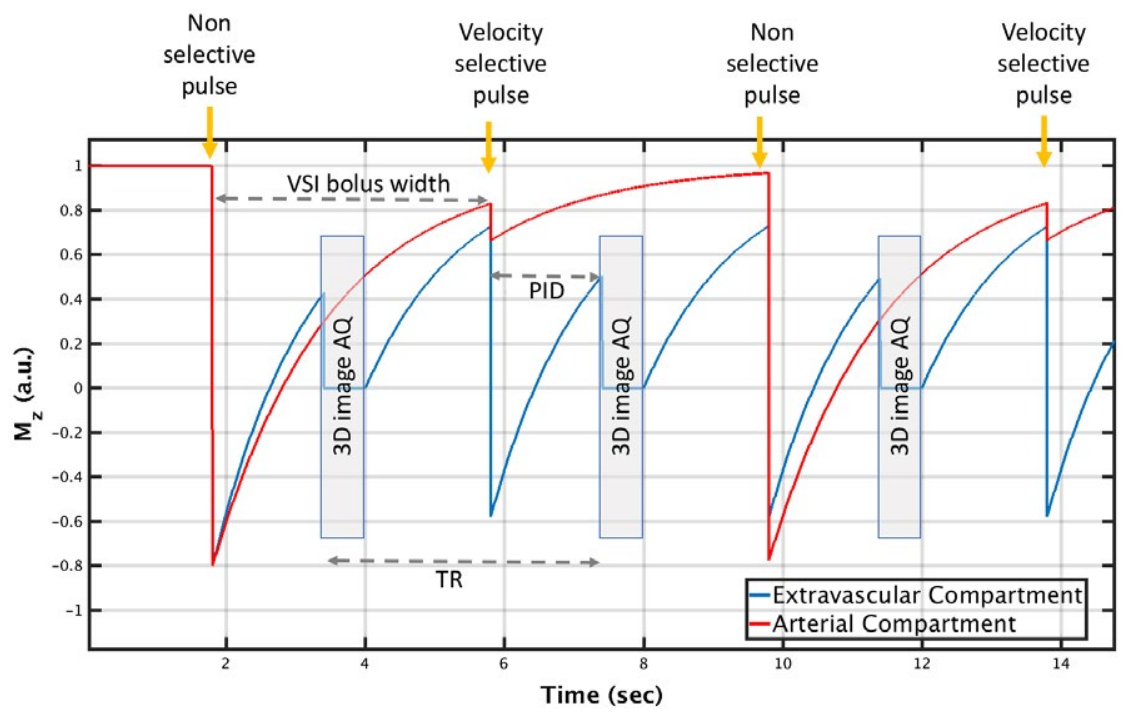

F I G URE 6 Bloch equation simulation of the evolution of the longitudinal magnetization in a perfusion based FMRI experiment using VSI pulses. No perfusion changes were simulated in this segment

\section{Optimization of VSI Time Series Timing Parameters}

\section{No Arterial Suppression \\ (Both compartments)}
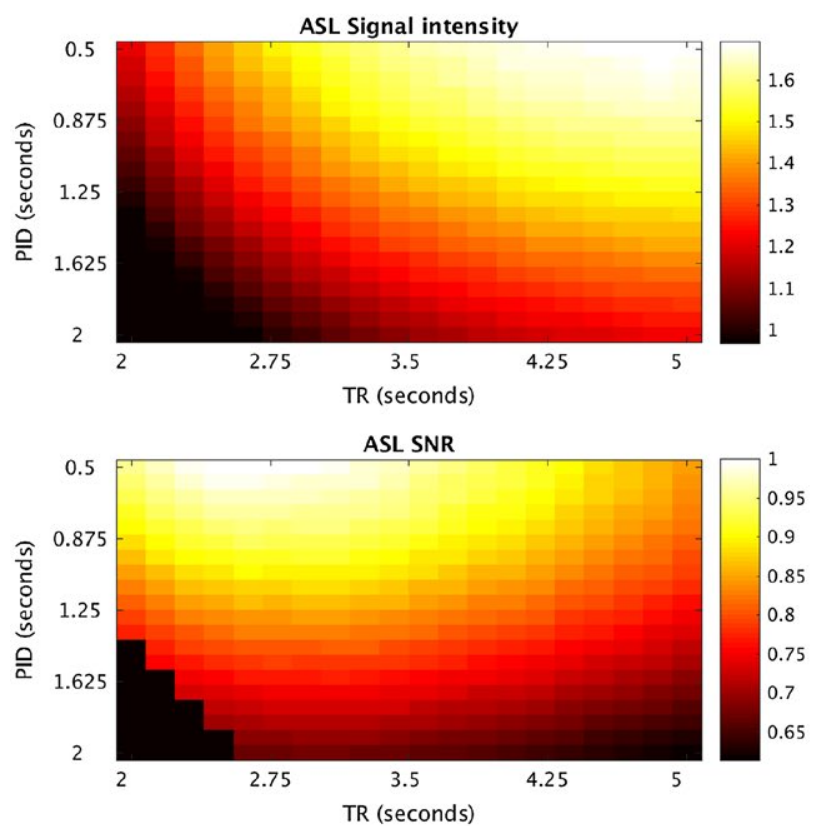

Arterial Suppression

(Extravascular Compartment Only)
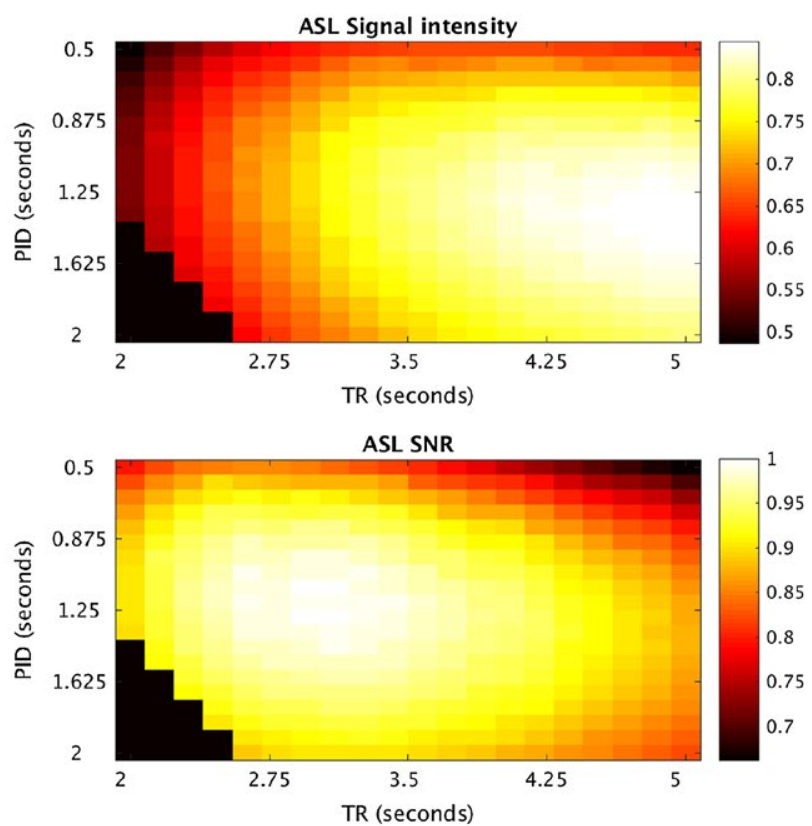

F I G U R E 7 Optimization of the VSI pulse sequence for FMRI. The top two panels show the simulated percent signal change observed without (left) and with (right) arterial suppression over a range of combinations of TR and PID. The bottom two panels show the theoretical SNR of the measurement, by scaling the ASL signal by the square root of the number of images that can be collected in that unit of time for each combination of parameters. (Note the black region on the bottom left corner of the plots. Those combinations of TR and PID are not realizable, so they are set to zero in the plot)

pulses are used). We also point out that the black regions on the bottom left corner of the images correspond to unrealistic timing combinations (i.e., TR $<$ PID + acquisition time) and were not included in the analysis. These simulations predict that, under ideal assumed conditions, optimal SNR efficiency occurs at $\mathrm{TR}=2.75 \mathrm{~s}$ and PID of $0.5 \mathrm{~s}$ when no arterial suppression is used. The optimal timing occurs at TR $=3.0 \mathrm{~s}$ and PID $=1.4 \mathrm{~s}$ when the arterial compartment is perfectly suppressed. 


\section{2 | FMRI results}

Activation maps (thresholded at $\mathrm{Z}>3$, or $P<0.0013$ ) from a representative subject collected with the PCASL sequence $(\mathrm{TR}=4 \mathrm{~s})$ and with the VSI sequence $(\mathrm{TR}=4 \mathrm{~s})$ are shown in Figure 8 overlaid on their corresponding perfusion weighted image (expressed as percent signal change between velocity selective and nonselective scans). The cases for $\mathrm{TR}=3$ and $2 \mathrm{~s}$ are shown in Supporting Information Figure S5. It is clear from the figure that VSI pulses produced larger \% signal changes than the PCASL images for our chosen timing parameters, approximated from the simulations on Figure 7. Table 2 show the temporal and spatial SNR of the VSI acquisitions, as well as the number of active voxels and the mean $\mathrm{Z}$ score of the active region (all quantities are expressed as a fraction relative to PCASL). The measures are expressed as a fraction relative to the PCASL case. Generally, the performance of VSI pulses was comparable to that of PCASL pulses at the same TR, except for subject 3, where PCASL outperformed VSI. However, the temporal SNR of the VSI pulse sequence was significantly larger (more than twice as much). When TR was reduced, VSI pulses outperformed the PCASL sequence. More specifically, the perfusion weighted images collected using the VSI labeling scheme showed significant improvement in temporal SNR relative to PCASL, and increased the $\mathrm{Z}$ scores in the active regions significantly.

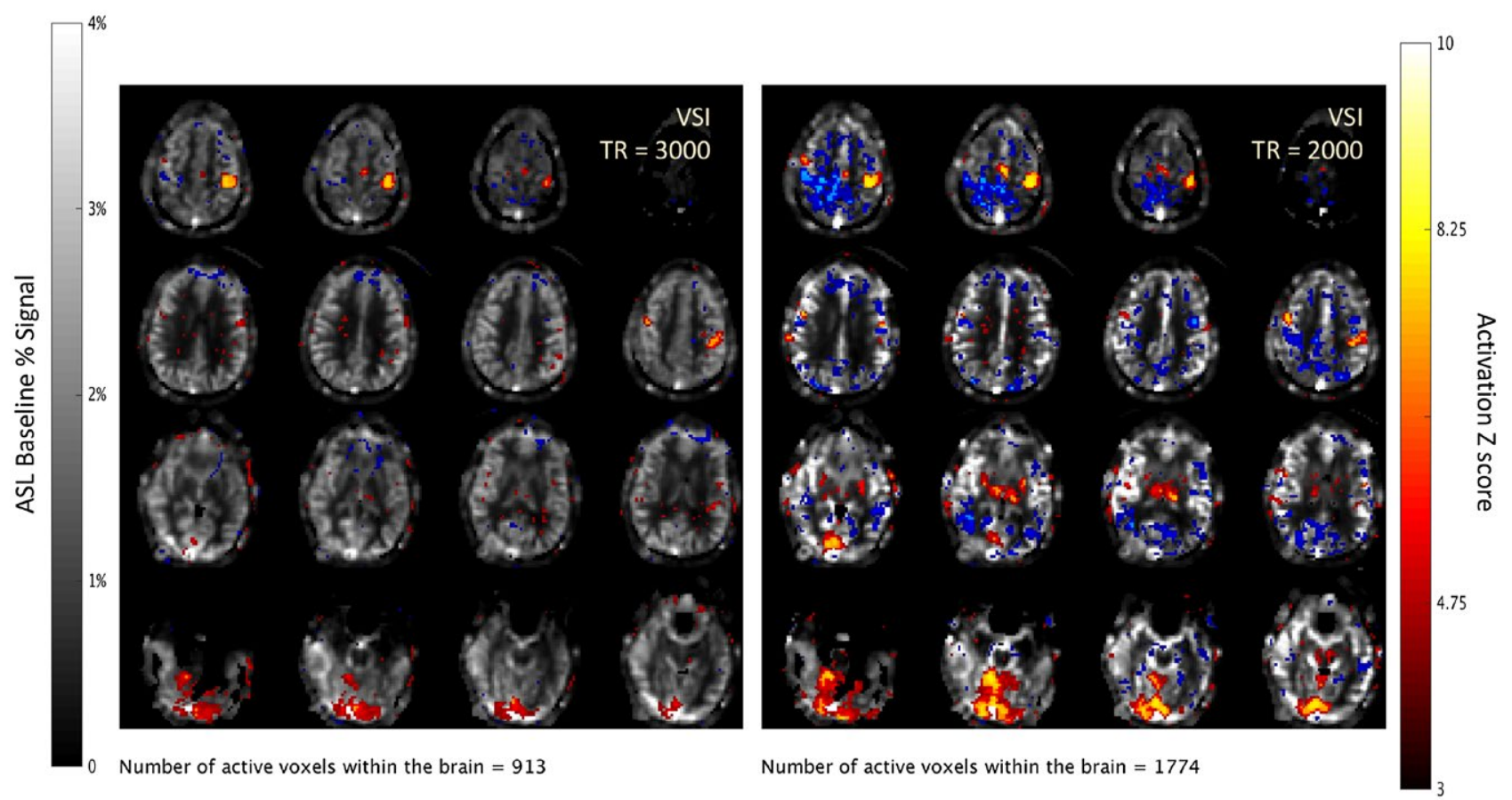

F I G URE 8 Activation maps from a representative subject overlaid on the mean perfusion image for FMRI experiments conducted using the PCASL sequence (for reference) and the VSI sequence using TR $=4$. The cases where TR was shortened to 3 and 2 s. are shown in Supporting Information Figure S5

TA B L E 2 Activation results obtained from VSI sequences, expressed as a fraction relative to PCASL. (Mean \pm S over subjects). The significance of the difference was assessed as the $P$-value of a paired t-test of the difference between each acquisition and PCASL. Asterisks indicate that the difference is statistically significant at $P<0.05$.

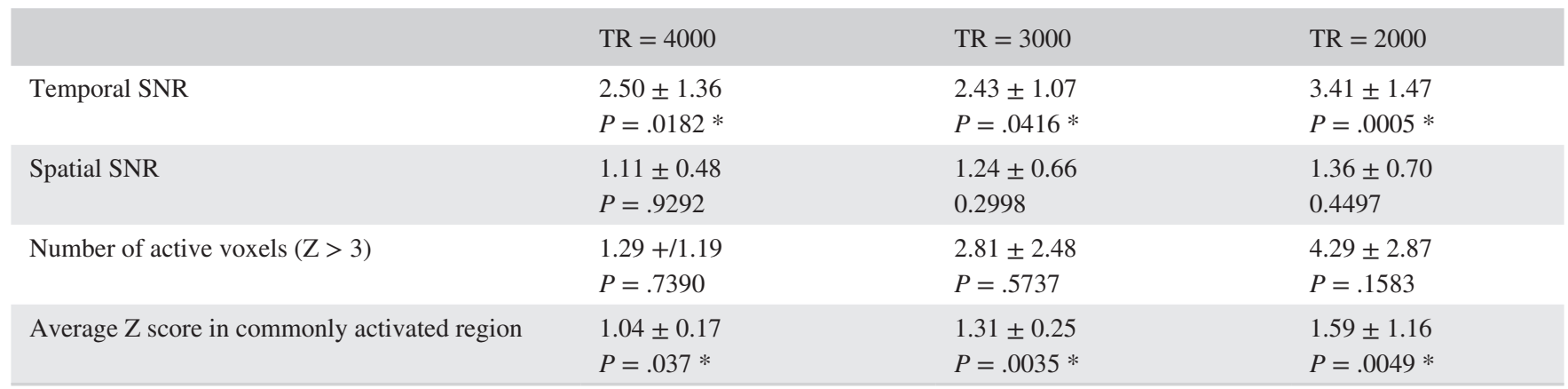

*indicates statistical significance. 
However, the table also shows that the spatial resolution and the number of voxels activated did not increase significantly in any of the VSI acquisitions. We observed that, when the post inversion delay was reduced to $1.2 \mathrm{~s}(\mathrm{TR}=2 \mathrm{~s})$, significant contribution from the vasculature was evident in the images. ASL images collected with the VSI sequence using a TR of $2 \mathrm{~s}$ failed to produce quality maps in subject 1 , and was excluded from the analysis.

\section{DISCUSSION}

The VSI sequence with careful choice of pulse sequence timing parameters, has superior SNR relative to PCASL because VSI pulses nearly eliminate the transit time from the application of the label and its arrival at the tissue. This reduces the loss of label from longitudinal relaxation quite significantly. Hence, VSI pulses are particularly well suited for white matter perfusion imaging, given the longer bolus arrival times of the white matter.

The kinetics of the VSI input function conforms to the standard two-compartment model and can be used to estimate important hemodynamic parameters. The fit to the model confirms the expected features of the technique and reveals important information about the input function. For example, short bolus arrival delays were observed in gray or white matter (less than half a second). We note that the bolus arrival time in white matter is longer than for gray matter, which is not unexpected, given the shape and velocity distribution of the brain's arterial network. Importantly, however, the size of the region where the VSI pulses are effective is not entirely clear, as it will be determined by the transmit coil's profile and the linear region of the gradients. Our data indicate that our 3T GE MR750 scanner's body coil produces a region that is large enough that the "tail-end" of the VSI arterial input function was not apparent in the explored time delay range (200-2600 ms). Beyond that time, the expected T1 decay of the label in a 3-s TR is approximately $85 \%$, so there is no need to apply crushers. However, it may be advantageous to use velocity selective saturation pulses to remove residual labeled arterial signals for shorter postlabeling delays and faster acquisitions. Future work will focus on characterizing the tail end of the arterial input function in greater detail.

There are some limitations in our modeling work that must be considered. The first is that the model includes a large number of parameters to be estimated, and we had to make some assumptions based on educated guesses to be able to estimate the parameters of interest. First, we assumed a Gaussian distribution of bolus arrival times at a given voxel, whose standard deviation was $100 \mathrm{~ms}$. Second, we fitted the labeling efficiency from the gray matter uptake curves and assumed that value as a constant for the white matter parameter fits, instead of estimating it separately, because the white matter fits were otherwise poor due to insufficient SNR. While the inversion efficiency of the blood should be the same for both gray and white matter (the VSI pulse was the same in both cases), it would have been preferable to estimate it separately for each compartment. These assumptions are sources of uncertainty in our parameter estimates.

Whereas we estimated perfusion in the tracer kinetic experiments in the first part of the study, we did not aim at quantification of cerebral blood flow (CBF) in the FMRI time series. Doing so would require collecting a reference scan as a measure of spin density. In our present experiment, the observed ASL FMRI signal amplitude roughly corresponds to a relative CBF measurement, but not an absolute one.

It must be noted that in our study, we did not make use of background suppression pulses to eliminate confounds from the comparison between the different input functions. The timing and number of background suppression pulses need to be optimized for each technique's characteristics and postlabeling delay. Thus, background suppression may not be equally effective in all techniques. However, the inversion of stationary spins inherent to the VSI method likely reduces the contribution of physiological fluctuations by reducing the contribution of stationary spins, which are not fully relaxed after the VSI pulses. Thus, the VSI pulse itself provides partial suppression of unwanted background signals.

Our FMRI study did not use arterial suppression pulses so the observed functional signal changes are due to a mix of arterial volume and perfusion changes. We made this choice because blood volume is known to increase significantly during activation, thus increasing the sensitivity to neural activity, although this results in some loss of spatial specificity, similar to vascular effects in the BOLD signal. We note that if greater specificity were needed, the arterial suppression pulses could indeed be included.

Given the features of the VSI's arterial input function, we observed that VSI pulses offer comparable SNR to PCASL for standard perfusion images, in line with previous findings. VSI timing parameters were chosen based on measurements of the label uptake curves, and on the above simulations (see Figures 6 and 7). It is important to note that the SNR would be higher with a much shorter postlabeling delay ( $<800 \mathrm{~ms})$, but those images would be dominated by the arterial compartment. Our simulations indicate that to measure the perfusion rate in the tissue, a longer delay is needed (>1200 ms).

We did not consider shorter timing parameters for PCASL, because that case has already been explored in the literature, as in the case of the AVAST technique. ${ }^{28-30}$

Another attractive feature of these particular VSI pulses with spin-echo refocusing is that they do not require off-resonance correction as in the case of PCASL. ${ }^{26,31}$ As noted by Qin et al, and in our own experience here, VSI pulses are robust to $\mathrm{B} 1$ and $\mathrm{B} 0$ imperfections but require calibration of the $\mathrm{B} 1$ pulse. 
VSI permits reduction of TR, which in turn allows for faster sampling of the perfusion time series, and thus more data collected in the same amount of time, which leads to further SNR efficiency and sensitivity gains. When the postlabeling delay is reduced along with TR, the contribution of the vasculature to the ASL signal increases, but this also increases the sensitivity to brain activity, consistent with previous work. ${ }^{28-30}$ However, we find that as TR gets shorter than 3 $\mathrm{s}$, the SNR did not improve in all subjects. This is most likely because the label may not have cleared the region completely between the label and control scans in cases where flow velocity in the arteries is slower, or the arterial network is more tortuous and the spins have a greater distance to travel. Our uptake curves suggest that the input function was longer than $2 \mathrm{~s}$, in which case, some of the spins may be labeled twice when the TR was $2 \mathrm{~s}$, which would muddle the quantification process and could potentially yield erroneous results. We hypothesize that this effect may be responsible for some of the spurious activation clusters in the figure.

A challenge with VSI-ASL is the presence of eddy current effects. As seen in Supporting Information Figure S6, our own phantom experiments show larger eddy current effects than those reported previously by Qin et al The eddy current artefacts observed on the edges of the phantom can reach $1 \%$ signal change or higher, which is problematic when the ASL signal itself is in that range. In this regard, there are three important observations to note. First, these artefacts correspond to scalp regions, rather than on the cortices, where the eddy current artefacts are much smaller. Second, the eddy current effects will bias the perfusion measurement, so perfusion values near the edges of the brain may be inflated. However, the bias from eddy currents does not interfere with the signal increases of interest in an FMRI experiment. Having said that, these eddy current artefacts are indeed a challenge for this technique that will require further development.

In summary, we have characterized the tracer kinetics of VSI pulses to design a perfusion based FMRI pulse sequence. Our data show that VSI-ASL is less sensitive to bolus arrival time effects, relative to PCASL. Additionally, it allows for faster temporal resolution, which can in turn be leveraged to achieve greater temporal SNR and increased sensitivity to perfusion changes due to activation.

\section{REFERENCES}

1. Alsop DC, Detre JA, Golay X, et al. Recommended implementation of arterial spin-labeled perfusion MRI for clinical applications: a consensus of the ISMRM Perfusion Study Group and the European Consortium for ASL in Dementia. Magn Reson Med. 2015;73(1):102-116.

2. Aguirre GK, Detre JA, Zarahn E, Alsop DC. Experimental design and the relative sensitivity of BOLD and perfusion fMRI. NeuroImage 2002; 15:488-500.
3. Aguirre GK, Detre JA, Wang J. Perfusion fMRI for functional neuroimaging. Int Rev Neurobiol. 2005;66:213-236.

4. Aguirre GK, Detre JA. The development and future of perfusion fMRI for dynamic imaging of human brain activity. Neuroimage. 2012;62(2):1279-1285.

5. Buch S, Ye Y, Haacke EM. Quantifying the changes in oxygen extraction fraction and cerebral activity caused by caffeine and acetazolamide. J Cereb Blood Flow Metab. 2017;37:825-836.

6. Pauls M, Clarke N, Trippier S, et al. Perfusion by arterial spin labelling following single dose tadalafil in small vessel disease (PASTIS): study protocol for a randomised controlled trial. Trials. 2017;18:229.

7. Hshieh TT, Dai W, Cavallari M, et al. Cerebral blood flow MRI in the nondemented elderly is not predictive of post-operative delirium but is correlated with cognitive performance. J Cereb Blood Flow Metab. 2017;37:1386-1397.

8. Demeter E, Hernandez-Garcia L, Sarter M, Lustig C. Challenges to attention: a continuous arterial spin labeling (ASL) study of the effects of distraction on sustained attention. NeuroImage. 2011;54:1518-1529.

9. Norris DG, Schwarzbauer C. Velocity selective radiofrequency pulse trains. J Magn Reson. 1999;137:231-236.

10. Wong EC, Cronin M, Wu W-C, Inglis B, Frank LR, Liu TT. Velocity-selective arterial spin labeling. Magn Reson Med. 2006;55(6):1334-1341.

11. Wu W-C, Wong EC. Feasibility of velocity selective arterial spin labeling in functional MRI. J Cereb Blood Flow Metab. 2007;27:831-838.

12. Schmid S, Heijtel D, Mutsaerts H, et al. Comparison of velocity- and acceleration-selective arterial spin labeling with [15O] $\mathrm{H} 2 \mathrm{O}$ positron emission tomography. J Cereb Blood Flow Metab. 2015;35:1296-1303.

13. Schmid S, Ghariq E, Teeuwisse WM, Webb A, Van Osch M. Acceleration-selective arterial spin labeling. Magn Reson Med. 2014;71:191-199.

14. Qin Q, vanZij1 P. Velocity-selective-inversion prepared arterial spin labeling. Magn Reson Med. 2016;76:1136-1148.

15. Qin Q, Van ZijlP. Velocity-selective inversion prepared arterial spin labeling for 3D whole-brain perfusion measurement. In: Proceedings of the 22nd Annual Meeting of ISMRM, Milan, Italy, 2014. Abstract 420.

16. Nielsen JF, Hernandez-Garcia L. Functional perfusion imaging using pseudocontinuous arterial spin labeling with lowflip-angle segmented 3D spiral readouts. Magn Reson Med. 2013;69:382-390.

17. Levitt MH, Freeman R, Frenkiel T. Broadband decoupling in high-resolution nuclear magnetic resonance spectroscopy. $A d v$ Magn Opt Reson. 1983;11:47-110.

18. Meakin JA, Jezzard P. An optimized velocity selective arterial spin labeling module with reduced eddy current sensitivity for improved perfusion quantification. Magn Reson Med. 2013;69:832-838.

19. Guo J, Meakin JA, Jezzard P, Wong EC. An optimized design to reduce eddy current sensitivity in velocity-selective arterial spin labeling using symmetric BIR-8 pulses. Magn Reson Med. 2015;73(3):1085-1094.

20. Qin Q, Grgac K, Van Zijl P. Determination of whole-brain oxygen extraction fractions by fast measurement of blood $\mathrm{T} 2$ in the jugular vein. Magn Reson Med. 2011;65:471-479. 
21. Williams DS, Detre JA, Leigh JS, Koretsky AP. Magnetic resonance imaging of perfusion using spin inversion of arterial water. Proc Natl Acad Sci U S A. 1992;89:212-216.

22. Detre JA, Leigh JS, Williams DS, Koretsky AP. Perfusion imaging. Magn Reson Med. 1992;23:37-45.

23. Lu H, Clingman C, Golay X, van Zijl P. Determining the longitudinal relaxation time (T1) of blood at 3.0 Tesla. Magn Reson Med. 2004;52:679-682.

24. Qin Q, Shin T, Schär M, Guo H, Chen H, Qiao Y. Velocity-selective magnetization-prepared non-contrast-enhanced cerebral MR angiography at 3 Tesla: improved immunity to B0/B1 inhomogeneity. Magn Reson Med. 2016;75:1232-1241.

25. Glover GH. FBIRN Stability phantom QA procedures. https:// docplayer.net/81514980-Fbirn-stability-phantom-qa-procedures. html. Accessed July 13, 2018.

26. Jahanian H, Noll DC, Hernandez-Garcia L. B0 field inhomogeneity considerations in pseudo-continuous arterial spin labeling (pCASL): effects on tagging efficiency and correction strategy. NMR Biomed. 2011;24:1202-1209.

27. Restom K, Behzadi Y, Liu TT. Physiological noise reduction for arterial spin labeling functional MRI. NeuroImage. 2006;31:1104-1115.

28. Jahanian H, Peltier S, Noll DC, Garcia LH. Arterial cerebral blood volume-weighted functional MRI using pseudocontinuous arterial spin tagging (AVAST). Magn Reson Med. 2015;73(3):1053-1106.

29. Liu P, Dimitrov I, Andrews T, et al. Multisite evaluations of a T2-relaxation-under-spin-tagging (TRUST) MRI technique to measure brain oxygenation. Magn Reson Med. 2016;75:680-687.

30. Shah YS, Hernandez-Garcia L, Jahanian H, Peltier SJ. Support vector machine classification of arterial volume-weighted arterial spin tagging images. Brain Behav. 2016;6(12):e00549.

31. Luh WM, Talagala SL, Li TQ, Bandettini PA. Pseudo-continuous arterial spin labeling at $7 \mathrm{~T}$ for human brain: estimation and correction for off-resonance effects using a Prescan. Magn Reson Med. 2013;69:402-410.

\section{SUPPORTING INFORMATION}

Additional Supporting Information may be found online in the supporting information tab for this article.

FIGURE S1 Velocity selective inversion pulse. The velocity selective gradient (played along the $\mathrm{z}$-axis) can be seen in the top panel. The middle panel shows the amplitude of the RF pulse, which consists of nine short pulses ( $20^{\circ} \mathrm{flip}$ ), interspersed with pairs of larger $180^{\circ}$ hard pulses. The bottom panel shows the phase of the RF pulse.
FIGURE S2 Diagram of the BIR-8 velocity selective saturation pulses proposed in Meakin and Jezzard and Guo et al. ${ }^{18,19}$

FIGURE S3 Velocity Profiles for the velocity selective inversion pulse used for labeling (left panel), and for the velocity selective saturation pulse used for arterial suppression (right panel). The first zero-crossing for the velocity saturation pulse happens at $0.7 \mathrm{~cm} / \mathrm{s}$. The efficiency of the velocity selective inversion pulse varies with velocity. According to this simulation, the mean labeling efficiency (defined as the magnetization difference between velocity selective and nonselective cases, relative to the relaxed magnetization) is $84 \%$ for the range between 5 and $80 \mathrm{~cm} / \mathrm{s}$.

FIGURE S4 Subtraction images collected at different times after the application of a VSI pulse. The images are computed as \% signal difference between selective and nonselective cases, relative to a reference image without any preparation pulses. Each column shows four slices from the collected volume. Each row represents a single slice collected at different post inversion delays. The images were acquired with using using a BIR-8 velocity selective saturation pulse for vascular suppression. These results are consistent with Figure 2.

FIGURE S5 Activation maps from a representative subject overlaid on the mean perfusion image for FMRI experiments conducted the VSI sequence using TR of 3 and $2 \mathrm{~s}$.

FIGURE S6 Eddy current tests carried out on an Agar phantom. Some differences in magnitude and phase are detected between the velocity selective and the nonselective cases. As expected, these differences decay with the post inversion delay, but contribute to the error in the early times of the uptake curve, primarily on the edges of the head.

How to cite this article: Hernandez-Garcia L,

Nielsen J-F, Noll DC. Improved sensitivity and temporal resolution in perfusion FMRI using velocity selective inversion ASL. Magn Reson Med. 2019;81: 1004-1015. https://doi.org/10.1002/mrm.27461 